\title{
Fractional Langevin equation with $\alpha$-stable noise. A link to fractional ARIMA time series
}

by

\author{
M. Magdziarz and A. Weron (Wrocław)
}

\begin{abstract}
We introduce a fractional Langevin equation with $\alpha$-stable noise and show that its solution $\left\{Y_{\kappa}(t), t \geq 0\right\}$ is the stationary $\alpha$-stable Ornstein-Uhlenbeck-type process recently studied by Taqqu and Wolpert. We examine the asymptotic dependence structure of $Y_{\kappa}(t)$ via the measure of its codependence $r\left(\theta_{1}, \theta_{2}, t\right)$. We prove that $Y_{\kappa}(t)$ is not a longmemory process in the sense of $r\left(\theta_{1}, \theta_{2}, t\right)$. However, we find two natural continuous-time analogues of fractional ARIMA time series with long memory in the framework of the Langevin equation.
\end{abstract}

1. Introduction. In recent years the concept of fractional differentiation and integration has aroused a wide interest in diverse scientific disciplines. Applications of the fractional calculus encompass many different fields, including stochastic processes, where the fractional differential equations turned out to be a very useful tool to derive processes with many desired properties $[1,2,4]$.

In what follows, we employ the techniques coming from the fractional calculus to obtain in an elegant way the fractional Ornstein-Uhlenbeck (O-U) $\alpha$-stable process, introduced in the recent paper by M. S. Taqqu and R. L. Wolpert [16, Sec. 4.2.2]. They define the fractional O-U $\alpha$-stable process in the following way: For $0<\alpha \leq 2$ let $L_{\alpha}(t)$ be the standard $\alpha$-stable Lévy motion [5, 15]. For $t \geq 0$ and $\lambda>0$ construct a series of processes indexed by $\kappa$ via the recursive recipe: $Y_{1}(t):=\sqrt{2 \lambda} \int_{-\infty}^{t} e^{-\lambda(t-s)} L_{\alpha}(d s), Y_{2}(t):=$ $\int_{-\infty}^{t} \lambda e^{-\lambda(t-s)} Y_{1}(s) d s$ and, in general, $Y_{\kappa}(t):=\int_{-\infty}^{t} \lambda e^{-\lambda(t-s)} Y_{\kappa-1}(s) d s$, which by the Fubini theorem for stochastic integrals (see [16] for a detailed discussion) gives

$$
Y_{\kappa}(t)=\frac{\sqrt{2 \lambda} \lambda^{\kappa-1}}{\Gamma(\kappa)} \int_{-\infty}^{t}(t-s)^{\kappa-1} e^{-\lambda(t-s)} L_{\alpha}(d s) .
$$

2000 Mathematics Subject Classification: 60G10, 60G52, 60H10, 62M10.

Key words and phrases: long memory, fractional Langevin equation, $\alpha$-stable processes, Ornstein-Uhlenbeck process, fractional ARIMA time series. 
For arbitrary positive $\kappa$ such that $\kappa>1-1 / \alpha$, equation (1) is taken in [16] as the definition of the fractional O-U $\alpha$-stable process. Note that for $\kappa=1$ we get the standard $\alpha$-stable O-U process $[5,15]$.

In Section 2 we show that the process (1) can be obtained as the solution of the so-called fractional Langevin equation with $\alpha$-stable noise. Next, in Section 3, we investigate its asymptotic behavior by means of the measure of codependence defined for a stationary process $Y(t)$ as

$$
\begin{aligned}
r\left(\theta_{1}, \theta_{2}, t\right)= & E\left[\exp \left\{i\left(\theta_{1} Y(t)+\theta_{2} Y(0)\right)\right\}\right] \\
& -E\left[\exp \left\{i \theta_{1} Y(t)\right\}\right] E\left[\exp \left\{i \theta_{2} Y(0)\right\}\right], \quad \theta_{1}, \theta_{2} \in \mathbb{R} .
\end{aligned}
$$

The function $r\left(\theta_{1}, \theta_{2}, t\right)$ has been shown to be the proper tool for describing the dependence structure of $\alpha$-stable processes [5-7, 9, 10, 15]. The asymptotic properties of log-fractional stable noise in terms of $r\left(\theta_{1}, \theta_{2}, t\right)$ were also investigated in the recent paper [8]. We employ the following definition [9] of long-range dependence for $\alpha$-stable processes: An $\alpha$-stable stationary process $Y(t)$ is said to have long memory if its codependence $r\left(\theta_{1}, \theta_{2}, t\right)$ satisfies

$$
\sum_{n=0}^{\infty}\left|r\left(\theta_{1}, \theta_{2}, n\right)\right|=\infty .
$$

Since for $\alpha$-stable moving average processes the function $r(1,-1, t)$ and the codifference

$$
\tau(t)=\ln E \exp \{i(Y(t)-Y(0))\}-\ln E \exp \{i Y(t)\}-\ln E \exp \{-i Y(0)\}
$$

are asymptotically equal as $t \rightarrow \infty$, one can also use $\tau(t)$ instead of $r\left(\theta_{1}, \theta_{2}, t\right)$ in (3) to characterize long memory of $Y(t)$ (see [7]).

The terms "long memory", "strong dependence" and "long-range dependence" are used interchangeably in the literature [3, 4]. They refer to phenomena in which the events that are arbitrarily distant still influence each other exceptionally strongly.

In Section 3 (Theorem 1) we give a negative answer to the question: does process (1) have the long-memory property in the sense of (3)? Our results complete the recent ones presented in [9] and [10], where the authors examine the dependence structure and the long-memory property of stationary processes which are generalizations of the classical $\alpha$-stable Ornstein-Uhlenbeck process.

Two different processes with long memory has long been known: fractional Brownian motion (A. N. Kolmogorov 1940) and fractional ARIMA (FARIMA) time series (C. W. J. Granger and R. Joyeux 1980); see [3, 4] and references therein. In Section 4 we investigate the relationship between these two models. The main finding is a construction of a continuous-time analogue of FARIMA time series in the framework of Langevin-type equations. We prove in Theorem 2 that the constructed process, similarly to FARIMA, displays long-range dependence. 
2. Fractional Langevin equation. Let us first recall the definition of the Bessel fractional derivative. Samko et al. [14] introduce the modified Bessel operator as

$$
G_{\lambda}^{\alpha} f(t)=\frac{1}{\Gamma(\alpha)} \int_{-\infty}^{t}(t-s)^{\alpha-1} e^{-\lambda(t-s)} f(s) d s .
$$

Here, $f \in L^{p}(\mathbb{R}, d s), 1 \leq p \leq \infty$. The Bessel fractional derivative is now introduced [14] as the operator inverse to $G_{\lambda}^{\alpha}$ on $L^{p}(\mathbb{R}, d s), 1 \leq p \leq \infty$,

$$
\left(\lambda I+\frac{d}{d t}\right)^{\alpha} f:=\left(G_{\lambda}^{\alpha}\right)^{-1} f
$$

where $I$ is the identity operator.

From the definition (4) we deduce that the fractional O-U $\alpha$-stable process (1) is equal (up to a constant) to the modified Bessel operator of the $\alpha$-stable noise $l_{\alpha}(t)$. Recall [2] that $l_{\alpha}(t)$ has $\alpha$-stable marginal distributions, its probability distribution is translation invariant and $l_{\alpha}(s)$ and $l_{\alpha}(t)$ are independent for $s \neq t$. Formally, $l_{\alpha}(s) d s=d L_{\alpha}(s)$, where $L_{\alpha}(s)$ is the standard $\alpha$-stable Lévy motion. We have

$$
G_{\lambda}^{\kappa} l_{\alpha}(t)=\frac{1}{\Gamma(\kappa)} \int_{-\infty}^{t}(t-s)^{\kappa-1} e^{-\lambda(t-s)} l_{\alpha}(s) d s .
$$

Heuristically, putting the Bessel fractional derivative on both sides of the above equation, we get

$$
\left(\lambda I+\frac{d}{d t}\right)^{\kappa} Y(t)=l_{\alpha}(t)
$$

where $\lambda, \kappa>0$ and

$$
Y(t)=\frac{1}{\Gamma(\kappa)} \int_{-\infty}^{t}(t-s)^{\kappa-1} e^{-\lambda(t-s)} L_{\alpha}(d s), \quad t \geq 0 .
$$

We call equation (5) the fractional Langevin equation and interpret the above process $Y(t)$ as its solution. Note that for $\kappa=1$, equation (5) becomes the standard $\alpha$-stable Langevin equation and its stationary solution is the $\mathrm{O}-\mathrm{U}$ process.

Concluding the above considerations, the solution of the fractional Langevin equation (5) is equal (up to a constant) to the fractional O-U $\alpha$-stable process (1). Thus, the standard techniques developed in fractional calculus allow us to obtain the stochastic process $Y_{\kappa}(t)$ in an elegant and coherent way. It should be noted that it is possible to consider a more general situation, when the $\alpha$-stable noise $l_{\alpha}(t)$ in the Langevin equation (5) is replaced by the Lévy noise (see [2]). In this case exactly the same procedure leads to a wide class of fractional O-U Lévy processes discussed in [16]. 
In the Gaussian case, when $\alpha=2, Y(t)$ is evidently a stationary centered process with normal marginal distributions and covariance function given for $t \geq 0$ by

$$
\begin{aligned}
c(t) & =E[Y(t) Y(0)] \\
& =\frac{1}{(\Gamma(\kappa))^{2}} \int_{-\infty}^{0}(|t|-s)^{\kappa-1} e^{-\lambda(|t|-s)}(-s)^{\kappa-1} e^{\lambda s} d s \\
& =\frac{1}{\Gamma(\kappa) \sqrt{\pi}(2 \lambda)^{\kappa-1 / 2}}|t|^{\kappa-1 / 2} K_{\kappa-1 / 2}(\lambda|t|),
\end{aligned}
$$

where $K_{v}(t)$ is the modified Bessel function of the second kind (see [14]). Since $K_{v}(t)$ decays exponentially fast, we deduce that in this particular case the process (6) (and equivalently (1)) does not have long memory. In the next section we examine the long-memory property for all $0<\alpha<2$.

3. Asymptotic dependence structure. In the next theorem we give precise formulas for the asymptotic behavior of the codependence $r\left(\theta_{1}, \theta_{2}, t\right)$ of the fractional O-U $\alpha$-stable process (1). We exclude the case $\theta_{1} \theta_{2}=0$, since then we have $r\left(\theta_{1}, \theta_{2}, t\right)=0$.

Theorem 1. Let $0<\alpha<2, \lambda>0, \kappa>0$ and $\kappa>1-1 / \alpha$. Then the codependence $r\left(\theta_{1}, \theta_{2}, t\right)$ of $Y_{\kappa}(t)$ satisfies:

(a) if $0<\alpha<1$, then

$$
r\left(\theta_{1}, \theta_{2}, t\right) \sim c_{\alpha}(\lambda, \kappa) C_{\alpha}\left(\theta_{1}, \theta_{2}\right)\left|\theta_{1}\right|^{\alpha} \frac{1}{\lambda \alpha} t^{\alpha(\kappa-1)} e^{-\lambda \alpha t},
$$

(b) if $\alpha=1$ and $\theta_{1} \theta_{2}>0$, then $r\left(\theta_{1}, \theta_{2}, t\right)=0$,

(c) if $\alpha=1$ and $\theta_{1} \theta_{2}<0$, then

$$
r\left(\theta_{1}, \theta_{2}, t\right) \sim 2 c_{1}(\lambda, \kappa) C_{1}\left(\theta_{1}, \theta_{2}\right)\left|\theta_{1}\right| \frac{1}{\lambda} t^{\kappa-1} e^{-\lambda t},
$$

(d) if $1<\alpha<2$, then

$$
r\left(\theta_{1}, \theta_{2}, t\right) \sim c_{\alpha}(\lambda, \kappa) d_{\alpha}(\lambda, \kappa) C_{\alpha}\left(\theta_{1}, \theta_{2}\right) \theta_{1} \frac{\left|\theta_{2}\right|^{\alpha}}{\theta_{2}} t^{\kappa-1} e^{-\lambda t}
$$

as $t \rightarrow \infty$. Here

$$
\begin{aligned}
c_{\alpha}(\lambda, \kappa) & =\left(\frac{\sqrt{2 \lambda} \lambda^{\kappa-1}}{\Gamma(\kappa)}\right)^{\alpha}, \\
C_{\alpha}\left(\theta_{1}, \theta_{2}\right) & =\exp \left\{-c_{\alpha}(\lambda, \kappa)\left(\left|\theta_{1}\right|^{\alpha}+\left|\theta_{2}\right|^{\alpha}\right) \frac{\Gamma(1+\alpha(\kappa-1))}{(\lambda \alpha)^{1+\alpha(\kappa-1)}}\right\}, \\
d_{\alpha}(\lambda, \kappa) & =-\alpha \frac{\Gamma((\kappa-1)(\alpha-1)+1)}{(\lambda \alpha)^{(\kappa-1)(\alpha-1)+1}} .
\end{aligned}
$$


Consequently, the fractional $O-U$-stable process $Y_{\kappa}(t)$ does not have the long-memory property in the sense of (3).

Proof. Put

$$
\begin{aligned}
I\left(\theta_{1}, \theta_{2}, t\right):= & -\ln E\left[\exp \left\{i\left(\theta_{1} Y_{\kappa}(t)+\theta_{2} Y_{\kappa}(0)\right)\right\}\right] \\
& +\ln E\left[\exp \left\{i \theta_{1} Y_{\kappa}(t)\right\}\right]+\ln E\left[\exp \left\{i \theta_{2} Y_{\kappa}(0)\right\}\right] .
\end{aligned}
$$

Note that for a symmetric $\alpha$-stable process the measure $-I(1,-1, t)$ is equal to the codifference $\tau(t)$. We have $r\left(\theta_{1}, \theta_{2}, t\right)=C_{\alpha}\left(\theta_{1}, \theta_{2}\right)\left(e^{-I\left(\theta_{1}, \theta_{2}, t\right)}-1\right)$ with

$$
\begin{aligned}
C_{\alpha}\left(\theta_{1}, \theta_{2}\right) & =E\left[\exp \left\{i \theta_{1} Y_{\kappa}(t)\right\}\right] E\left[\exp \left\{i \theta_{2} Y_{\kappa}(0)\right\}\right] \\
& =\exp \left\{-c_{\alpha}(\lambda, \kappa)\left(\left|\theta_{1}\right|^{\alpha}+\left|\theta_{2}\right|^{\alpha}\right) \frac{\Gamma(1+\alpha(\kappa-1))}{(\lambda \alpha)^{1+\alpha(\kappa-1)}}\right\},
\end{aligned}
$$

where in the last equality we used the following formula [15, p. 122]:

$$
E\left[\exp \left\{i \theta \int_{\mathbb{R}} f(x) L_{\alpha}(d x)\right\}\right]=\exp \left\{-|\theta|^{\alpha} \int_{\mathbb{R}}|f(x)|^{\alpha} d x\right\}
$$

with $f \in L^{\alpha}(\mathbb{R}, d x)$. Thus, if $I\left(\theta_{1}, \theta_{2}, t\right) \rightarrow 0$ as $t \rightarrow \infty$ then

$$
r\left(\theta_{1}, \theta_{2}, t\right) \sim-C_{\alpha}\left(\theta_{1}, \theta_{2}\right) I\left(\theta_{1}, \theta_{2}, t\right),
$$

which indicates that $r(\cdot)$ and $I(\cdot)$ are asymptotically equal. Recall that $f(t) \sim$ $g(t)$ if and only if $f(t) / g(t) \rightarrow 1$ as $t \rightarrow \infty$.

Equation (9) and some standard calculations yield

$$
I\left(\theta_{1}, \theta_{2}, t\right)=c_{\alpha}(\lambda, \kappa)\left(\int_{0}^{\infty} I_{1}(t, s) d s+\int_{0}^{\infty} I_{2}(t, s) d s\right),
$$

where

$$
\begin{aligned}
& I_{1}(t, s)=-\left|\theta_{1}\right|^{\alpha}(t+s)^{\alpha(\kappa-1)} e^{-\lambda \alpha(t+s)} \\
& I_{2}(t, s)=\left|\theta_{1}(t+s)^{\kappa-1} e^{-\lambda(t+s)}+\theta_{2} s^{\kappa-1} e^{-\lambda s}\right|^{\alpha}-\left|\theta_{2}\right|^{\alpha} s^{\alpha(\kappa-1)} e^{-\lambda \alpha s}
\end{aligned}
$$

and $c_{\alpha}(\lambda, \kappa)$ is given by $(7)$.

(a) For every $s \in(0, \infty)$ we get

$$
e^{\lambda \alpha t} t^{-\alpha(\kappa-1)} I_{1}(t, s) \rightarrow-\left|\theta_{1}\right|^{\alpha} e^{-\lambda \alpha s} \quad \text { as } t \rightarrow \infty
$$

and

$$
\begin{aligned}
\sup _{t>1} \mid e^{\lambda \alpha t} t^{-\alpha(\kappa-1)} & I_{1}(t, s) \mid \\
& \leq \begin{cases}\left|\theta_{1}\right|^{\alpha} e^{-\lambda \alpha s} & \text { if }-1<\alpha(\kappa-1) \leq 0, \\
\left|\theta_{1}\right|^{\alpha}(1+s)^{\alpha(\kappa-1)} e^{-\lambda \alpha s} & \text { if } \alpha(\kappa-1)>0,\end{cases}
\end{aligned}
$$

which belongs to $L^{1}((0, \infty), d s)$. Thus from the dominated convergence 
theorem,

$$
\int_{0}^{\infty} I_{1}(t, s) d s \sim-\left|\theta_{1}\right|^{\alpha} e^{-\lambda \alpha t} t^{\alpha(\kappa-1)} \int_{0}^{\infty} e^{-\lambda \alpha s} d s=-\left|\theta_{1}\right|^{\alpha} e^{-\lambda \alpha t} t^{\alpha(\kappa-1)} \frac{1}{\lambda \alpha}
$$

as $t \rightarrow \infty$. Further, for every $s \in(0, \infty), e^{\lambda \alpha t} t^{-\alpha(\kappa-1)} I_{2}(t, s) \rightarrow 0$ as $t \rightarrow \infty$, and since $\left.|| a\right|^{\alpha}-|b|^{\alpha}|\leq| a-\left.b\right|^{\alpha}$ for $\alpha \in(0,1], a, b \in \mathbb{R}$, we have $\left|I_{2}(t, s)\right| \leq$ $\left|I_{1}(t, s)\right|$, and consequently

$$
e^{\lambda \alpha t} t^{-\alpha(\kappa-1)} \int_{0}^{\infty} I_{2}(t, s) d s \rightarrow 0 \quad \text { as } t \rightarrow \infty .
$$

Finally, from (13) and (14) we conclude

$$
I\left(\theta_{1}, \theta_{2}, t\right) \sim-c_{\alpha}(\lambda, \kappa)\left|\theta_{1}\right|^{\alpha} \frac{1}{\lambda \alpha} t^{\alpha(\kappa-1)} e^{-\lambda \alpha t} \quad \text { as } t \rightarrow \infty
$$

(b) Equation (11) for $\alpha=1$ and $\theta_{1} \theta_{2}>0$ gives $I_{1}(t, s)+I_{2}(t, s)=0$ and therefore $r\left(\theta_{1}, \theta_{2}, t\right)=0$.

(c) For $\alpha=1$ and $\theta_{1} \theta_{2}<0$ we have, for every $s \in(0, \infty)$,

$$
e^{\lambda t} t^{-(\kappa-1)}\left(I_{1}(t, s)+I_{2}(t, s)\right) \rightarrow-2\left|\theta_{1}\right| e^{-\lambda s} \quad \text { as } t \rightarrow \infty
$$

and we show in a similar manner as in part (a) that

$$
I\left(\theta_{1}, \theta_{2}, t\right) \sim-2 c_{1}(\lambda, \kappa)\left|\theta_{1}\right| \frac{1}{\lambda} t^{\kappa-1} e^{-\lambda t} \quad \text { as } t \rightarrow \infty .
$$

(d) Recall the decomposition (11). For $1<\alpha<2$ and every $s \in(0, \infty)$ we get $e^{\lambda t} t^{-(\kappa-1)} I_{1}(t, s) \rightarrow 0$ as $t \rightarrow \infty$ and since

$$
\sup _{t>1}\left|e^{\lambda t} t^{-(\kappa-1)} I_{1}(t, s)\right| \leq c_{1}\left|\theta_{1}\right|^{\alpha} e^{-\lambda s}
$$

( $c_{1}$ is a constant depending only on $\alpha, \lambda$ and $\kappa$ ), the dominated convergence theorem yields

$$
e^{\lambda t} t^{-(\kappa-1)} \int_{0}^{\infty} I_{1}(t, s) d s \rightarrow 0 \quad \text { as } t \rightarrow \infty
$$

Furthermore, for $s \in(0, \infty)$,

$$
e^{\lambda t} t^{-(\kappa-1)} I_{2}(t, s) \rightarrow \alpha \theta_{1} \frac{\left|\theta_{2}\right|^{\alpha}}{\theta_{2}} e^{-\lambda \alpha s} s^{(\kappa-1)(\alpha-1)} \quad \text { as } t \rightarrow \infty .
$$

Taking advantage of the inequalities (see [10]): ||$a-\left.b\right|^{\alpha}-b^{\alpha} \mid \leq a^{\alpha}+\alpha a b^{\alpha-1}$ and ||$a+\left.b\right|^{\alpha}-b^{\alpha} \mid \leq a^{\alpha}+\alpha a b^{\alpha-1}$, valid for $a, b \geq 0$ and $\alpha \in(1,2]$, we obtain

$$
\begin{aligned}
& \sup _{t>1}\left|e^{\lambda t} t^{-(\kappa-1)} I_{2}(t, s)\right| \\
& \quad \leq \begin{cases}c_{1}\left|\theta_{1}\right|^{\alpha} e^{-\lambda s}+\alpha\left|\theta_{1}\right|\left|\theta_{2}\right|^{\alpha-1} e^{-\lambda \alpha s} s^{(\kappa-1)(\alpha-1)} & \text { if } \kappa-1 \leq 0, \\
c_{2}\left|\theta_{1}\right|^{\alpha} e^{-\lambda s}+\alpha\left|\theta_{1}\right|\left|\theta_{2}\right|^{\alpha-1} e^{-\lambda \alpha s} s^{(\kappa-1)(\alpha-1)}(1+s)^{\kappa-1} & \text { if } \kappa-1>0,\end{cases}
\end{aligned}
$$


which belongs to $L^{1}((0, \infty), d s)$. Thus, the dominated convergence theorem yields

$$
e^{\lambda t} t^{-(\kappa-1)} \int_{0}^{\infty} I_{2}(t, s) d s \rightarrow \alpha \theta_{1} \frac{\left|\theta_{2}\right|^{\alpha}}{\theta_{2}} \int_{0}^{\infty} e^{-\lambda \alpha s} s^{(\kappa-1)(\alpha-1)} d s \quad \text { as } t \rightarrow \infty .
$$

Since

$$
\int_{0}^{\infty} e^{-\lambda \alpha s} s^{(\kappa-1)(\alpha-1)} d s=\frac{\Gamma((\kappa-1)(\alpha-1)+1)}{(\lambda \alpha)^{(\kappa-1)(\alpha-1)+1}}
$$

we obtain

$$
I\left(\theta_{1}, \theta_{2}, t\right) \sim-c_{\alpha}(\lambda, \kappa) d_{\alpha}(\lambda, \kappa) \theta_{1} \frac{\left|\theta_{2}\right|^{\alpha}}{\theta_{2}} t^{\kappa-1} e^{-\lambda t} \quad \text { as } t \rightarrow \infty,
$$

and the proof is complete.

The above result shows that the lack of long memory observed in the Gaussian case $(\alpha=2)$ also occurs in the more general stable case for all $\alpha \in$ $(0,2)$. A similar relationship can be found in other cited works [9, 10], which suggests that the long-memory property for the stationary $\alpha$-stable processes with integral representation $Y(t)=\int_{\mathbb{R}} f(t, s) L_{\alpha}(d s)$ is determined only by the kernel function $f$. The kernel can be represented explicitly by means of the tools used in ergodic theory $[12,13]$. An interesting open problem is to characterize the long-range dependence property of the process $Y(t)$ in terms of its kernel.

4. Relationship with FARIMA models. The FARIMA (fractional autoregressive integrated moving average) discrete time processes have found widespread acceptance as mathematical models for various empirical time series with long memory $([3,4]$ and references therein). We begin by recalling the definition.

Let $B$ be the shift operator defined by $B X(t)=X(t-1)$ and $\Delta$ be the difference operator, i.e. $\Delta X(t)=X(t)-X(t-1)=(I-B) X(t)$. The FARIMA model is a generalization of the classical $\operatorname{ARIMA}(p, \kappa, q)$ process

$$
\Phi(B) \Delta^{\kappa} X(t)=\Theta(B) \varepsilon_{t}, \quad t \in \mathbb{N} .
$$

Here $\Phi$ and $\Theta$ are polynomials of degree $p$ and $q$ respectively, $\varepsilon_{n}$ are assumed to be i.i.d. symmetric $\alpha$-stable random variables, and $\kappa$ is a non-negative integer. Now, for $\operatorname{FARIMA}(p, \kappa, q)$ the parameter $\kappa$ is allowed to take also fractional values, either positive or negative. To avoid unnecessary complications, in our further discussion we set $p=q=0$. Then the $\operatorname{FARIMA}(0, \kappa, 0)$ model is the solution of

$$
\Delta^{\kappa} X(t)=\varepsilon_{t}
$$


Consequently, $X(t)=\Delta^{-\kappa} \varepsilon_{t}$, where the operator $\Delta^{-\kappa}=(1-B)^{-\kappa}$ for the fractional parameter $\kappa$ is formally interpreted via the Taylor expansion of the function $(1-z)^{-\kappa}=\sum_{j=0}^{\infty} b_{j}(-\kappa) z^{j}$. The coefficients in the series are

$$
b_{j}(-\kappa)=\frac{\Gamma(j+\kappa)}{\Gamma(\kappa) \Gamma(j+1)} .
$$

Thus, the formal definition of a $\operatorname{FARIMA}(0, \kappa, 0)$ process is

$$
X(t)=\Delta^{-\kappa} \varepsilon_{t}=(1-B)^{-\kappa} \varepsilon_{t}=\sum_{j=0}^{\infty} b_{j}(-\kappa) \varepsilon_{t-j}, \quad t \in \mathbb{N} .
$$

$X(t)$ is a stationary moving average process and a necessary condition for the series (17) to converge a.s. is $-\infty<\kappa<1-1 / \alpha$. In the Gaussian case, i.e. when $\alpha=2$, the rate of decay of the covariance function $\operatorname{Cov}(t):=$ $E[X(t) X(0)]-E[X(t)] E[X(0)]$ for the FARIMA model is $t^{2 \kappa-1}$, which shows that for $\kappa \geq 0$ we have $\sum_{n=0}^{\infty}|\operatorname{Cov}(n)|=\infty$ and $X(t)$ is a process with longrange dependence. For $\alpha<2$ the covariance does not exist and one has to employ other measures of dependence, appropriate for stochastic processes with infinite second moment. In [6] the authors determine the asymptotic behavior of the codifference $\tau(t)$ for $\operatorname{FARIMA}(p, \kappa, q)$. In particular, they prove that if $X(t)$ is a $\operatorname{FARIMA}(0, \kappa, 0)$ process with symmetric $\alpha$-stable innovations, $0<\alpha \leq 2$ and $\kappa$ is not an integer, then the following two cases can hold. If either (i) $\alpha \leq 1$ or (ii) $\alpha>1$ and $(\alpha-1)(\kappa-1)>-1$, then

$$
\lim _{t \rightarrow \infty} \frac{\tau(t)}{t^{\alpha(\kappa-1)+1}}=\frac{1}{[\Gamma(\kappa)]^{\alpha}} \int_{0}^{\infty} g(x) d x
$$

where

$$
g(x)=x^{(\kappa-1) \alpha}+(1+x)^{(\kappa-1) \alpha}-\left(x^{\kappa-1}-(1+x)^{\kappa-1}\right)^{\alpha} .
$$

If $\alpha>1$ and $(\alpha-1)(\kappa-1)<-1$, then

$$
\lim _{t \rightarrow \infty} \frac{\tau(t)}{t^{\kappa-1}}=\frac{\alpha}{\Gamma(\kappa)} \sum_{j=0}^{\infty} b_{j}(-\kappa)^{\langle\alpha-1\rangle},
$$

where $\langle z\rangle=|z| \operatorname{sign}(z)$. As a consequence, for $\kappa>1-2 / \alpha$ a $\operatorname{FARIMA}(0, \kappa, 0)$ process has long memory in the sense of (3).

The question arises whether one can find a stationary $\alpha$-stable process with continuous time $t$, which could be regarded as an appropriate counterpart of $\operatorname{FARIMA}(0, \kappa, 0)$ in the sense of the dependence structure. We propose the following continuous-time process:

$$
Z_{1}(t):=\frac{1}{\Gamma(\kappa)} \int_{-\infty}^{t}(t-s)^{\kappa-1} L_{\alpha}(d s), \quad t \in \mathbb{R}_{+},
$$


as a counterpart of FARIMA. To justify this choice, first note that from Stirling's formula for the Gamma function, $\Gamma(z) \sim e^{-z} z^{z-1 / 2} \sqrt{2 \pi}$ as $z \rightarrow \infty$, we get the asymptotic behavior of the coefficients (16),

$$
b_{j}(-\kappa)=\frac{\Gamma(j+\kappa)}{\Gamma(\kappa) \Gamma(j+1)} \sim \frac{j^{\kappa-1}}{\Gamma(\kappa)} \quad \text { as } j \rightarrow \infty .
$$

Therefore, the FARIMA process (17) can be considered as an approximate sum for a stochastic integral,

$$
\begin{aligned}
X(t) & =\sum_{j=0}^{\infty} b_{j}(-\kappa) \varepsilon_{t-j}=\sum_{j=-\infty}^{t} b_{t-j}(-\kappa) \varepsilon_{j} \\
& =\sum_{j=-\infty}^{t} \int_{j-1}^{j} b_{t-j}(-\kappa) \mathbf{1}_{(j-1, j]}(s) L_{\alpha}(d s) \\
& \approx \frac{1}{\Gamma(\kappa)} \int_{-\infty}^{t}(t-s)^{\kappa-1} L_{\alpha}(d s)=Z_{1}(t) .
\end{aligned}
$$

On the other hand, the FARIMA equation (15) can be replaced by its continuous-time counterpart, namely

$$
\frac{d^{\kappa}}{d t^{\kappa}} Z_{1}(t)=l_{\alpha}(t), \quad t \in \mathbb{R}_{+}
$$

where the difference operator $\Delta$ is replaced by the fractional derivative operator $d^{\kappa} / d t^{\kappa}$ (see [14]), and the sequence of i.i.d. variables $\varepsilon_{t}$ is replaced by the $\alpha$-stable Lévy noise $l_{\alpha}(t)$. Here, the fractional derivative $d^{\kappa} / d t^{\kappa}$ is defined as the inverse to the integral operator

$$
I^{\kappa} f(t)=\frac{1}{\Gamma(\kappa)} \int_{-\infty}^{t}(t-s)^{\kappa-1} f(s) d s, \quad \kappa>0 .
$$

Therefore, the process $Z_{1}(t)$ can be interpreted as the solution of the FARIMAtype equation (19).

The main problem in the case under consideration is that the kernel function in representation (18) does not belong to $L^{\alpha}(\mathbb{R}, d s)$, and therefore the integral is divergent. The first method of avoiding this difficulty is to replace (19) by the fractional Langevin equation (5) introduced in Section 2 and let $\lambda \searrow 0$. However, the solution of (5) is well defined only for $\kappa>$ $1-1 / \alpha$. Let us remind the reader that for FARIMA processes exactly the opposite condition for $\kappa$ is required, which undoubtedly causes difficulties while comparing the properties of the solution of (5) and FARIMA. Below, we present two different ways to get rid of the divergence of the integral in (18). 
4.1. Linear fractional stable noise. The first idea is to introduce an increment process of $Z_{1}(t)$. We define formally

$$
\begin{aligned}
Z_{2}(t) & :=Z_{1}(t+1)-Z_{1}(t) \\
& =\frac{1}{\Gamma(\kappa)} \int_{\mathbb{R}}\left[(t+1-s)_{+}^{\kappa-1}-(t-s)_{+}^{\kappa-1}\right] L_{\alpha}(d s),
\end{aligned}
$$

where $a_{+}=\max \{a, 0\}$. Now, we see that for $\kappa \in(1-1 / \alpha, 2-1 / \alpha)$ the kernel function in (20) is $\alpha$-integrable, since it behaves like $s^{\kappa-1}$ as $s \rightarrow 0$ and like $s^{\kappa-2}$ as $s \rightarrow \infty$. Let us emphasize that $Z_{2}(t)$ should be considered as an "approximation" of the increments of the $\operatorname{FARIMA}(0, \kappa, 0)$ model, and not as the process related directly to FARIMA. Putting $\kappa-1=H-1 / \alpha$ we see that $Z_{2}(t)$ is a version of the well-known linear fractional stable noise $l_{\alpha, H}(t)$ (see $[5,15])$. The process $l_{\alpha, H}(t)$ is a classical example of a long-memory $\alpha$-stable process. It is defined as the increment process of the $H$-self-similar linear fractional stable motion $L_{\alpha, H}(t)$ (i.e. $\left.l_{\alpha, H}(t)=L_{\alpha, H}(t+1)-L_{\alpha, H}(t)\right)$. Therefore, the process $Z_{2}(t)$, which is regarded as an "approximation" of the increments of FARIMA $(0, \kappa, 0)$, is a version of $l_{\alpha, H}(t)$. Thus, as a by-product of our considerations, we obtain a link between two significant long-memory processes, namely the linear fractional stable noise can be considered as an "approximation" of the increments of FARIMA, which confirms that in both models the property of long-range dependence has the same origin. Additionally, the relationship between the parameters of $Z_{2}(t)$ and $l_{\alpha, H}(t)$ is $\kappa-1=H-1 / \alpha$. The importance of this relationship for $H$-self-similar models driven by $\alpha$-stable noise in physics and related areas has long been known $[11,17]$.

4.2. Continuous-time FARIMA equation. The second possibility is to perturb the solution $Z_{1}(t)$ of the FARIMA-type equation (19) in order to get rid of the possible divergence of the integral at the origin. Note that in the first case considered, application of the fractional Langevin equation allowed us to avoid divergence of the integral at $\infty$. For $\varepsilon>0$ and $t \geq 0$ we define

$$
Z_{3}(t):=\frac{1}{\Gamma(\kappa)} \int_{-\infty}^{t}(t-s+\varepsilon)^{\kappa-1} L_{\alpha}(d s)
$$

$Z_{3}(t)$ is a stationary moving average process. It is well defined for $\kappa<1-1 / \alpha$, which, in contrast with the first two cases studied, agrees exactly with the permissible range of the parameter $\kappa$ for FARIMA. Since the behavior of the codifference $\tau(t)$ for FARIMA is well known (see [6] and the beginning of Section 4), to compare the dependence structure of FARIMA and $Z_{3}(t)$, we determine in the next theorem the asymptotics of the codifference corresponding to $Z_{3}(t)$. 
Theorem 2. Let $0<\alpha \leq 2$ and $-\infty<\kappa<1-1 / \alpha$. Then the codifference $\tau(t)$ of $Z_{3}(t)$ satisfies:

(a) If either (i) $\alpha \leq 1$ or (ii) $\alpha>1$ and $(\alpha-1)(\kappa-1)>-1$, then

$$
\lim _{t \rightarrow \infty} \frac{\tau(t)}{t^{\alpha(\kappa-1)+1}}=\frac{1}{[\Gamma(\kappa)]^{\alpha}} \int_{0}^{\infty} g(x) d x
$$

where

$$
g(x)=x^{(\kappa-1) \alpha}+(1+x)^{(\kappa-1) \alpha}-\left(x^{\kappa-1}-(1+x)^{\kappa-1}\right)^{\alpha} .
$$

(b) If $\alpha>1$ and $(\alpha-1)(\kappa-1)<-1$, then

$$
\lim _{t \rightarrow \infty} \frac{\tau(t)}{t^{\kappa-1}}=\frac{\alpha}{\Gamma(\kappa)} \int_{0}^{\infty} h(x) d x
$$

where

$$
h(x)=\frac{(x+\varepsilon)^{(\kappa-1)(\alpha-1)}}{[\Gamma(\kappa)]^{\alpha-1}} .
$$

Consequently, for $\kappa>1-2 / \alpha$ the process $Z_{3}(t)$ has long memory in the sense of (3).

Proof. (a) Since

$$
\begin{aligned}
\tau(t)= & \ln E\left[\exp \left\{i\left(Z_{3}(t)-Z_{3}(0)\right)\right\}\right] \\
& -\ln E\left[\exp \left\{i Z_{3}(t)\right\}\right]-\ln E\left[\exp \left\{-i Z_{3}(0)\right\},\right.
\end{aligned}
$$

formula (9) and some standard calculations give

$$
\begin{aligned}
\tau(t)=\frac{1}{[\Gamma(\kappa)]^{\alpha}} \int_{0}^{\infty}\left[(x+\varepsilon)^{(\kappa-1) \alpha}+(t+x+\varepsilon)^{(\kappa-1) \alpha}\right. \\
\left.-\left((x+\varepsilon)^{\kappa-1}-(t+x+\varepsilon)^{\kappa-1}\right)^{\alpha}\right] d x .
\end{aligned}
$$

After the change of variables $x \mapsto t x$ we get

$$
\tau(t)=\frac{t^{\alpha(\kappa-1)+1}}{[\Gamma(\kappa)]^{\alpha}} \int_{0}^{\infty}\left[\left|a_{t}(x)\right|^{\alpha}+\left|b_{t}(x)\right|^{\alpha}-\left|a_{t}(x)-b_{t}(x)\right|^{\alpha}\right] d x,
$$

where $a_{t}(x)=(x+\varepsilon / t)^{\kappa-1}$ and $b_{t}(x)=(1+x+\varepsilon / t)^{\kappa-1}$. Thus for fixed $x \in(0, \infty)$ we have

$$
a_{t}(x) \rightarrow x^{\kappa-1} \quad \text { and } \quad b_{t}(x) \rightarrow(1+x)^{\kappa-1} \quad \text { as } t \rightarrow \infty .
$$

To apply the dominated convergence theorem, we need the following inequality (see [9]): for $r, s \in \mathbb{R}$,

$$
|| r+\left.s\right|^{\alpha}-|r|^{\alpha}-|s|^{\alpha} \mid \leq \begin{cases}2|r|^{\alpha} & \text { if } 0<\alpha \leq 1 \\ (\alpha+1)|r|^{\alpha}+\alpha|r||s|^{\alpha-1} & \text { if } 1<\alpha \leq 2 .\end{cases}
$$


Using this result we obtain

$$
\begin{aligned}
\left.\sup _{t>1}|| a_{t}(x)\right|^{\alpha}+\left|b_{t}(x)\right|^{\alpha}-\left|a_{t}(x)-b_{t}(x)\right|^{\alpha} \mid & \\
& \leq \begin{cases}2|1+x|^{\alpha(\kappa-1)} & \text { if } 0<\alpha \leq 1, \\
(\alpha+1)|1+x|^{\alpha(\kappa-1)}+\alpha|1+x|^{\kappa-1}|x|^{(\alpha-1)(\kappa-1)} & \text { if } 1<\alpha \leq 2,\end{cases}
\end{aligned}
$$

which in both cases belongs to $L^{1}((0, \infty), d s)$ (note that in the second case we assumed $(\alpha-1)(\kappa-1)>-1)$. Thus the dominated convergence theorem yields

$$
\lim _{t \rightarrow \infty} \frac{\tau(t)}{t^{\alpha(\kappa-1)+1}}=\frac{1}{[\Gamma(\kappa)]^{\alpha}} \int_{0}^{\infty} g(x) d x
$$

where $g(x)=x^{(\kappa-1) \alpha}+(1+x)^{(\kappa-1) \alpha}-\left(x^{\kappa-1}-(1+x)^{\kappa-1}\right)^{\alpha}$.

(b) From (22) we get

$$
\tau(t)=\frac{1}{[\Gamma(\kappa)]^{\alpha}} \int_{0}^{\infty}\left[(p(x))^{\alpha}+\left(q_{t}(x)\right)^{\alpha}-\left(p(x)-q_{t}(x)\right)^{\alpha}\right] d x,
$$

where $p(x)=(x+\varepsilon)^{\kappa-1}$ and $q_{t}(x)=(t+x+\varepsilon)^{\kappa-1}$. Note that for fixed $x \in(0, \infty)$ we have $q_{t}(x) \sim t^{\kappa-1}$ as $t \rightarrow \infty$. From the mean-value theorem,

$$
f(r+s)-f(r)=s \int_{0}^{1} f^{\prime}(r+u s) d u
$$

where $f$ is an appropriately smooth function, we obtain

$$
[p(x)]^{\alpha}-\left[p(x)-q_{t}(x)\right]^{\alpha}=\alpha q_{t}(x) \int_{0}^{1}\left[p(x)-u q_{t}(x)\right]^{\alpha-1} d u
$$

and consequently $[p(x)]^{\alpha}-\left[p(x)-q_{t}(x)\right]^{\alpha} \sim \alpha t^{\kappa-1}[p(x)]^{\alpha-1}$ as $t \rightarrow \infty$. Moreover, $\left[q_{t}(x)\right]^{\alpha} / t^{\kappa-1} \rightarrow 0$ as $t \rightarrow 0$, since $\alpha>1$. Thus for fixed $x \in(0, \infty)$ we have

$$
\frac{[p(x)]^{\alpha}+\left[q_{t}(x)\right]^{\alpha}-\left[p(x)-q_{t}(x)\right]^{\alpha}}{t^{\kappa-1}} \rightarrow \alpha[p(x)]^{\alpha-1}=\alpha(x+\varepsilon)^{(\alpha-1)(\kappa-1)}
$$

as $t \rightarrow \infty$. To apply the dominated convergence theorem, we need the following inequality: for $r, s>0$ and $\alpha \in(1,2]$,

$$
r^{\alpha}+s^{\alpha}-|r-s|^{\alpha} \leq(\alpha+1) r s^{\alpha-1} \text {. }
$$

To prove (23), we consider two cases. First, let $r \geq s$. Define $f_{s}(r):=r^{\alpha}+$ $s^{\alpha}-|r-s|^{\alpha}-(\alpha+1) r s^{\alpha-1}$. We have to show that $f_{s}(r) \leq 0$. We have $f_{s}(0)=0$ and

$$
f_{s}^{\prime}(r)=\alpha r^{\alpha-1}-\alpha(r-s)^{\alpha-1}-(\alpha+1) s^{\alpha-1} \leq \alpha s^{\alpha-1}-(\alpha+1) s^{\alpha-1} \leq 0 .
$$


Thus $f_{s}(r) \leq 0$. Let now $r<s$. Using the mean-value theorem we get

$$
\begin{aligned}
r^{\alpha}+s^{\alpha}-(s-r)^{\alpha} & \leq r s^{\alpha-1}+\alpha r \int_{0}^{1}[(s-r)+r u]^{\alpha-1} d u \\
& \leq r s^{\alpha-1}+\alpha r s^{\alpha-1}=(\alpha+1) r s^{\alpha-1}
\end{aligned}
$$

which proves (23).

Now, using the above result we obtain

$$
\begin{aligned}
\sup _{t>1} \frac{\left|[p(x)]^{\alpha}+\left[q_{t}(x)\right]^{\alpha}-\left[p(x)-q_{t}(x)\right]\right|^{\alpha}}{t^{\kappa-1}} & \leq \sup _{t>1} \frac{(\alpha+1) q_{t}(x)[p(x)]^{\alpha-1}}{t^{\kappa-1}} \\
\leq(\alpha+1)[p(x)]^{\alpha-1} & =(\alpha+1)(x+\varepsilon)^{(\alpha-1)(\kappa-1)},
\end{aligned}
$$

which for $(\alpha-1)(\kappa-1)<-1$ belongs to $L^{1}((0, \infty), d s)$. Finally, the dominated convergence theorem yields

$$
\lim _{t \rightarrow \infty} \frac{\tau(t)}{t^{\kappa-1}}=\frac{\alpha}{\Gamma(\kappa)} \int_{0}^{\infty} h(x) d x,
$$

where

$$
h(x)=\frac{(x+\varepsilon)^{(\kappa-1)(\alpha-1)}}{[\Gamma(\kappa)]^{\alpha-1}} .
$$

To show that for $\kappa>1-2 / \alpha$ the process $Z_{3}(t)$ has long memory in the sense of (3), it is enough to observe that the condition $\kappa>1-2 / \alpha$ is equivalent to $\alpha(\kappa-1)+1>-1$. Therefore, for $\alpha \leq 1$ we have $\sum_{t=0}^{\infty}|\tau(t)|$ $=\infty$. For $\alpha \in(1,2], \kappa>1-2 / \alpha$ implies $(\alpha-1)(\kappa-1)>-1$, thus $\tau(t)$ $\sim c t^{\alpha(\kappa-1)+1}$ and $\sum_{t=0}^{\infty}|\tau(t)|=\infty$.

The above results for $Z_{3}(t)$ are actually identical with the ones for a FARIMA $(0, \kappa, 0)$ process [6]. The rate of convergence of $\tau(t)$ in both cases is exactly the same and does not depend on $\varepsilon$, which implies that both processes have the long-memory property for the same range of $\kappa$. The parameter $\varepsilon$ only affects the constant in part (b) of the theorem, whereas the constant in (a) is identical for both processes. For these reasons we may consider $Z_{3}(t)$ as a "proper" continuous-time counterpart of $\operatorname{FARIMA}(0, \kappa, 0)$ in the sense of the dependence structure, while, as shown by the considerations in the previous subsection, the increments of the linear fractional stable motion are the counterpart of the increments of a FARIMA process.

Acknowledgements. We would like to thank the anonymous referee for his constructive comments leading to improvement of the presentation. 


\section{References}

[1] V. V. Anh, C. C. Heyde and N. N. Leonenko, Dynamic models of long-memory processes driven by Lévy noise with applications to finance and macroeconomics, J. Appl. Probab. 39 (2002), 730-747.

[2] V. V. Anh and R. McVinish, Fractional differential equations driven by Lévy noise, J. Appl. Math. Stoch. Anal. 16 (2003), 97-119.

[3] J. Beran, Statistics for Long-Memory Processes, Chapman \& Hall, New York, 1994.

[4] P. Doukhan, G. Oppenheim and M. S. Taqqu (eds.), Theory and Applications of Long-Range Dependence, Birkhäuser, Boston, 2003.

[5] A. Janicki and A. Weron, Simulation and Chaotic Behaviour of $\alpha$-Stable Stochastic Processes, Dekker, New York, 1994.

[6] P. S. Kokoszka and M. S. Taqqu, Fractional ARIMA with stable innovations, Stochastic Process. Appl. 60 (1995), 19-47.

[7] J. B. Levy and M. S. Taqqu, A characterization of the asymptotic behavior of stationary stable processes, in: Stable Processes and Related Topics, S. Cambanis et al. (eds.), Birkhäuser, Boston, 1991, 181-198.

[8] -, - , The asymptotic codifference and covariation of log-fractional stable noise, preprint, 2005.

[9] M. Maejima and K. Yamamoto, Long-memory stable Ornstein-Uhlenbeck processes, Electronic J. Probab. 8 (2003), art. 19, 18 pp.

[10] M. Magdziarz, The dependence structure of the fractional Ornstein-Uhlenbeck process, Probab. Math. Statist. 25 (2005), 97-104.

[11] Sz. Mercik, K. Weron, K. Burnecki and A. Weron, Enigma of self-similarity of fractional Lévy stable motions, Acta Phys. Polon. B 34 (2003), 3773-3791.

[12] J. Rosinski, On the structure of stationary stable processes, Ann. Probab. 23 (1995), 1163-1187.

[13] - Decomposition of stationary $\alpha$-stable random fields, ibid. 28 (2001), 1797-1813.

[14] S. G. Samko, A. A. Kilbas and O. I. Marichev, Fractional Integrals and Derivatives: Theory and Applications, Gordon and Breach, London, 1993.

[15] G. Samorodnitsky and M. S. Taqqu, Stable Non-Gaussian Random Processes: Stochastic Models with Infinite Variance, Chapman \& Hall, New York, 1994.

[16] M. S. Taqqu and R. L. Wolpert, Fractional Ornstein-Uhlenbeck Lévy processes and the Telecom process: Upstairs and downstairs, Signal Process. 85 (2005), 1523-1545.

[17] A. Weron, K. Burnecki, Sz. Mercik and K. Weron, Complete description of all self-similar models driven by Lévy stable noise, Phys. Rev. E 71 (2005), 016113.

\section{Hugo Steinhaus Center}

Institute of Mathematics and Computer Science

Wrocław University of Technology

50-370 Wrocław, Poland

E-mail: marcin.magdziarz@pwr.wroc.pl

aleksander.weron@pwr.wroc.pl

Received April 11, 2006

Revised version April 27, 2007 